\title{
Constraining the Dark Universe
}

\author{
Rachel Bean ${ }^{\mathrm{a}}$, Steen H. Hansen ${ }^{\mathrm{b}}$, Alessandro Melchiorri ${ }^{\mathrm{b}}$ \\ aTheoretical Physics, The Blackett Laboratory, Imperial College, Prince Consort Road, London, U.K. \\ ${ }^{\mathrm{b}} \mathrm{NAPL}$, University of Oxford, Keble road, OX1 3RH, Oxford, UK
}

\begin{abstract}
We combine complementary datasets to constrain dark energy. Using standard Big Bang Nucleosynthesis and the observed abundances of primordial nuclides to put constraints on $\Omega_{Q}$ at temperatures near $T \sim 1 \mathrm{MeV}$, we find the strong constraint $\Omega_{Q}(\mathrm{MeV})<0.045$ at $2 \sigma$ c.l.. Under the assumption of flatness, using results from Cosmic Microwave Background (CMB) anisotropy measurements, high redshift supernovae (SN-Ia) observations and data from local cluster abundances we put a new constraint on the equation of state parameter $w_{Q}<-0.85$ at $68 \%$ c.l..
\end{abstract}

Introduction. The discovery that the universe's evolution may be dominated by an effective cosmological constant [1], is one of the most remarkable cosmological findings of recent years. An exceptional opportunity is now opening up to decipher the nature of dark matter [2, to test the veracity of theories and reconstruct the dark matters profile using a wide variety of observations over a broad redshift range.

One candidate that could possibly explain the observations is a dynamical scalar "quintessence" field. One of the strong aspects of quintessence theories is that they go some way explaining the fine-tuning problem, why the energy density producing the acceleration is $\sim 10^{-120} M_{p l}^{4}$. A vast range of "tracker" (see for example [3, 11]) and "scaling" (for example [12]-15]) quintessence models exist which approach attractor solutions, giving the required energy density, independent of initial conditions. The common characteristic of quintessence models is that their equations of state, $w_{Q}=p / \rho$, vary with time whilst a cosmological constant remains fixed at $w_{Q=\Lambda}=-1$. Observationally distinguishing a time variation in the equation of state or finding $w_{Q}$ different from -1 will therefore be a success for the quintessential scenario.

We will here discuss observational constraints on general quintessence models.

In [23 we used standard big bang nucleosynthesis and the observed abundances of primordial nuclides to put constraints on the amplitude of the energy density, $\Omega_{Q}$, at temperatures near $T \sim 1 \mathrm{MeV}$. The inclusion of a scaling field increases the expansion rate of the universe, and changes the ratio of neutrons to protons at freeze-out and hence the predicted abundances of light elements.

In [24] we have then combined the latest observations of the Cosmic Microwave Background (CMB) anisotropies provided by the Boomerang [16], DASI [17] and Maxima [18] experiments and the information from Large Scale Structure (LSS) with the luminosity distance of high redshift supernovae (SN-
Ia) to put constraints on the dark energy equation of state parameterized by a redshift independent quintessence-field pressure-to-density ratio $w_{Q}$. We also made use of the Hubble Space Telescope (HST) constraint on the Hubble parameter $h=0.72 \pm 0.08$ [19].

We will briefly review the results obtained in those paper in the next sections.

Early-Universe Constraints from BBN. In the last few years important experimental progress has been made in the measurement of light element primordial abundances. For the ${ }^{4} \mathrm{He}$ mass fraction, $Y_{\mathrm{He}}$, two marginally compatible measurements have been obtained from regression against zero metallicity in blue compact galaxies. A low value $Y_{\mathrm{He}}=0.234 \pm$ 0.003 [5] and a high one $Y_{\mathrm{He}}=0.244 \pm 0.002$ [6] give realistic bounds. We use the high value in our analysis; if one instead considered the low value, the bounds obtained would be even stronger.

Observations in different quasar absorption line systems give a relative abundance of deuterium, which is critical in fixing the baryon fraction, of $D / H=(3.0 \pm 0.4) \cdot 10^{-5}$ [7].

In the standard $\mathrm{BBN}$ scenario, the primordial abundances are a function of the baryon density $\eta \sim \Omega_{b} h^{2}$ only. In order to put constraints on the energy density of a primordial field a $T \sim \mathrm{MeV}$, we modified the standard BBN code [4] by including the quintessence energy component $\Omega_{Q}$. We then performed a likelihood analysis in the parameter space $\left(\Omega_{b} h^{2}, \Omega_{Q}^{B B N}\right)$ using the observed abundances $Y_{\mathrm{He}}$ and $D / H$. In Fig. 1 w we plot the 1,2 and $3 \sigma$ likelihood contours in the $\left(\Omega_{b} h^{2}, \Omega_{Q}^{B B N}\right)$ plane.

Our main result is that the experimental data for ${ }^{4} \mathrm{He}$ and $\mathrm{D}$ does not favour the presence of a dark energy component, providing the strong constraint $\Omega_{Q}(\mathrm{MeV})<0.045$ at $2 \sigma$ (corresponding to $\lambda>9$ for the exponential potential scenario), strengthening significantly the previous limit of $8,15, \Omega_{Q}(\mathrm{MeV})<$ 0.2 . The reason for the difference is due to the improvement in the measurements of the observed 


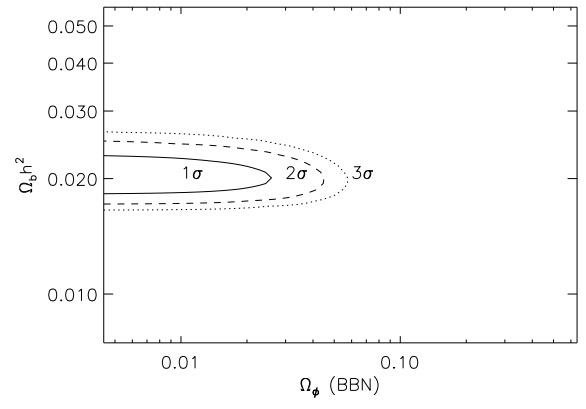

Figure 1. 1,2 and $3 \sigma$ likelihood contours in the $\left(\Omega_{b} h^{2}, \Omega_{Q}(1 \mathrm{MeV})\right)$ parameter space derived from ${ }^{4} \mathrm{He}$ and $\mathrm{D}$ abundances.

abundances, especially for the deuterium, which now corresponds to approximately $\Delta N_{\text {eff }}<0.2-0.3$ additional effective neutrinos (see, e.g. [9]), whereas Ref. 88.15] used the conservative value $\Delta N_{\text {eff }}<1.5$. One could worry about the effect of any underestimated systematic errors, and we therefore multiplied the error-bars of the observed abundances by a factor of 2. Even taking this into account, there is still a strong constraint $\Omega_{Q}(\mathrm{MeV})<0.09(\lambda>6.5)$ at $2 \sigma$.

Constraints on the Dark energy equation of state.

The importance of combining different data sets in order to obtain reliable constraints on $w_{Q}$ has been stressed by many authors (see e.g. [20], 21], 222]), since each dataset suffers from degeneracies between the various cosmological parameters and $w_{Q}$. Even if one restricts consideration to flat universes and to a value of $w_{Q}$ constant in time then the SN-Ia luminosity distance and position of the first $\mathrm{CMB}$ peak are highly degenerate in $w_{Q}$ and $\Omega_{Q}$, the energy density in quintessence.

The effects of varying $w_{Q}$ on the angular power spectrum of the CMB anisotropies can be reduced to just two . Firstly, since the inclusion of quintessence changes the overall content of matter and energy, the angular diameter distance of the acoustic horizon size at recombination will be altered. In flat models (i.e. where the energy density in matter is equal to $\left.\Omega_{M}=1-\Omega_{Q}\right)$, this creates a shift in the peaks positions of the angular spectrum as

$$
\begin{aligned}
\mathcal{R}= & \sqrt{\left(1-\Omega_{Q}\right)} y, \\
y= & \int_{0}^{z_{\operatorname{dec}}}\left[\left(1-\Omega_{Q}\right)(1+z)^{3}\right. \\
& \left.+\Omega_{Q}(1+z)^{3\left(1+w_{Q}\right)}\right]^{-1 / 2} d z .
\end{aligned}
$$

It is important to note that the effect is completely degenerate in the interplay between $w_{Q}$ and $\Omega_{Q}$. Furthermore, it does not add any new features beyond those produced by the presence of a cosmological constant [25], and it is not particularly sensitive to further time dependencies of $w_{Q}$.

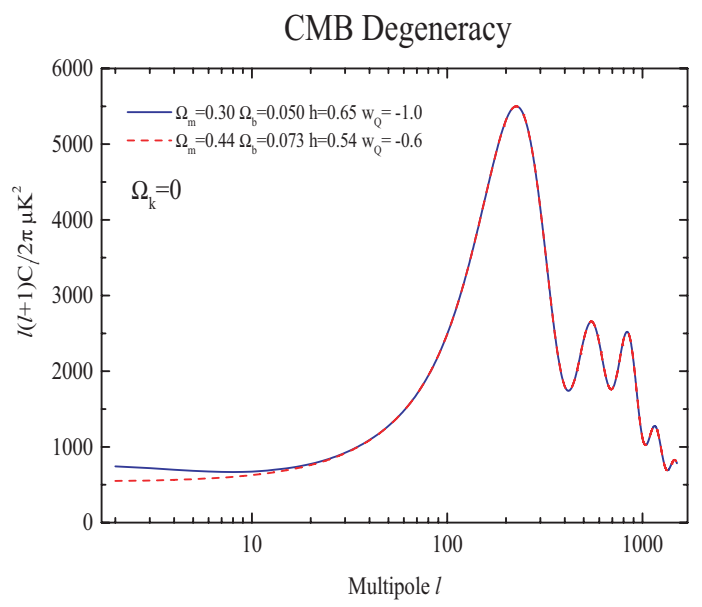

Figure 2. CMB power spectra and the angular diameter distance degeneracy. The models are computed assuming flatness, $\left.\Omega_{k}=1-\Omega_{M}-\Omega_{Q}=0\right)$. The Integrated Sachs Wolfe effect on large angular scale slightly breaks the degeneracy. The degeneracy can be broken with a strong prior on $h$, in this paper we use the results from the HST.

Secondly, the time-varying Newtonian potential after decoupling will produce anisotropies at large angular scales through the Integrated Sachs-Wolfe (ISW) effect. The curve in the CMB angular spectrum on large angular scales depends not only on the value of $w_{Q}$ but also its variation with redshift. However, this effect will be difficult to disentangle from the same effect generated by a cosmological constant, especially in view of the affect of cosmic variance and/or gravity waves on the large scale anisotropies.

In order to emphasize the importance of degeneracies between all these parameters while analyzing the CMB data, we plot in Figure 2 some degenerate spectra, obtained keeping the physical density in matter $\Omega_{M} h^{2}$, the physical density in baryons $\Omega_{b} h^{2}$ and $\mathcal{R}$ fixed. As we can see from the plot, models degenerate in $w_{Q}$ can easily be constructed. However the combination of CMB data with other different datasets can break the mentioned degeneracies.

Type Ia supernovae, in particular, can be extremely useful in this. Evidence that the universe's expansion rate was accelerating was first provided by 


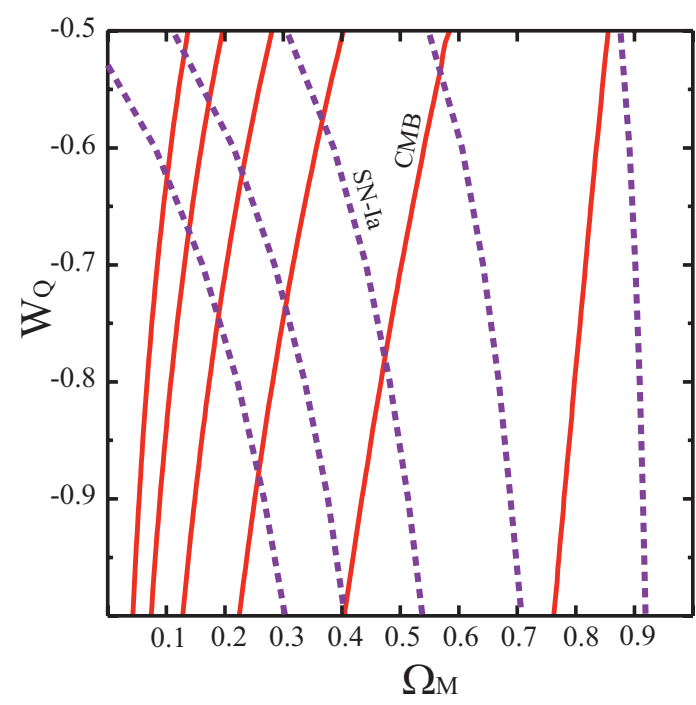

Figure 3. Contours of constant $\mathcal{R}(\mathrm{CMB})$ and $S N-I a$ luminosity distance in the $w_{Q}-\Omega_{M}$ plane. The degeneracy between the two distance measures can be broken by combining the two sets of complementary information. The luminosity distance is chosen to be equal to $d_{l}$ at $z=1$ for a fiducial model with $\Omega_{\Lambda}=0.7, \Omega_{M}=0.3, h=0.65$. (We note that as $\Omega_{Q}=1-\Omega_{M}$ goes to zero the dependence of $\mathcal{R}$ and $d_{L}$ upon $w_{Q}$ also become zero, as there is no dark energy present.) Figure taken from [24]

two groups, the SCP and High-Z Search Team([1]) using type Ia supernovae (SN-Ia) to probe the nearby expansion dynamics. SN-Ia seem to be good standard candles, as they exhibit a strong phenomenological correlation between the decline rate and peak magnitude of the luminosity. The observed apparent bolometric luminosity is related to the luminosity distance, measured in Mpc, by $m_{B}=M+$ $5 \log d_{L}(z)+25$, where $\mathrm{M}$ is the absolute bolometric magnitude. The luminosity distance is sensitive to the cosmological evolution through an integral dependence on the Hubble factor $d_{l}=(1+$ z) $\int_{0}^{z}\left(d z^{\prime} / H\left(z^{\prime}, \Omega_{Q}, w_{q}\right)\right.$, and can therefore be used to constrain the scalar equation of state. As can be seen in Figure 3 there is an inherent degeneracy in the luminosity distance in the $\Omega_{M} / w_{Q}$ plane; one can see that little can be said about the equation of state from luminosity distance data alone. However, the degeneracies of CMB and SN1a data complement one another so that together they offer a more powerful approach for constraining $w_{Q}$.

Table I shows the 1- $\sigma$ constraints on $w_{Q}$ for different combinations of priors, obtained in 24] af-
Table 1

Constraints on $w_{Q}$ and $\Omega_{M}=1-\Omega_{Q}$ using different priors and datasets. We always assume flatness and $t_{0}>10 \mathrm{Gyr}$. The $1 \sigma$ limits are found from the $16 \%$ and $84 \%$ integrals of the marginalized likelihood. The HST prior is $h=0.72 \pm 0.08$ while for the BBN prior we use the conservative bound $\Omega_{b} h^{2}=0.020 \pm 0.005$.

$$
\begin{array}{lr}
\text { CMB+HST } & w_{Q}<-0.62 \\
& 0.15<\Omega_{M}<0.45 \\
\text { CMB+HST+BBN } & -0.95<w_{Q}<-0.62 \\
& 0.15<\Omega_{M}<0.42 \\
\text { CMB+HST+SN-Ia } & -0.94<w_{Q}<-0.74 \\
\text { CMB+HST+SN-Ia+LSS } & 0.16<\Omega_{M}<0.34 \\
& w_{Q}<-0.85 \\
& 0.28<\Omega_{M}<0.43 \\
\hline
\end{array}
$$

ter marginalizing over all remaining nuisance parameters. The analysis is restricted to flat universes. One can see that $w_{Q}$ is poorly constrained from CMB data alone, even when the strong HST prior on the Hubble parameter, $h=0.72 \pm 0.08$, is assumed. Adding a Big Bang Nucleosynthesis prior, $\Omega_{b} h^{2}=0.020 \pm 0.005$, has small effect on the $\mathrm{CMB}+\mathrm{HST}$ result. Adding SN-Ia breaks the CMB $\Omega_{Q}-w_{Q}$ degeneracy and improves the upper limit on $w_{Q}$, with $w_{Q}<-0.74$. Finally, including information from local cluster abundances through $\sigma_{8}=(0.55 \pm 0.1) \Omega_{M}^{-0.5}$, where $\sigma_{8}$ is the rms mass fluctuation in spheres of $8 h^{-1} \mathrm{Mpc}$, further breaks the quintessential-degeneracy, giving $w_{Q}<-0.85$ at $1-\sigma$. Also reported in Table I, are the constraints on $\Omega_{M}$. As we can see, the combined data suggests the presence of dark energy with high significance, even in the case $\mathrm{CMB}+\mathrm{HST}$. It is interesting to project our likelihood in the $\Omega_{Q}-w_{Q}$ plane. Proceeding as in [27, we attribute a likelihood to a point in the $\left(\Omega_{M}\right.$, $w_{Q}$ ) plane by finding the remaining parameters that maximize it. We then define our $68 \%, 95 \%$ and $99 \%$ contours to be where the likelihood falls to $0.32,0.05$ and 0.01 of its peak value, as would be the case for a two dimensional multivariate Gaussian. In Figure 4 we plot likelihood contours in the $\left(\Omega_{M}, w_{Q}\right)$ plane for the joint analyses of $\mathrm{CMB}+\mathrm{SN}-\mathrm{Ia}+\mathrm{HST}+\mathrm{LSS}$ data together with the contours from the SN-Ia dataset only. As we can see, the combination of the data breaks the luminosity distance degeneracy.

\section{Conclusions.}




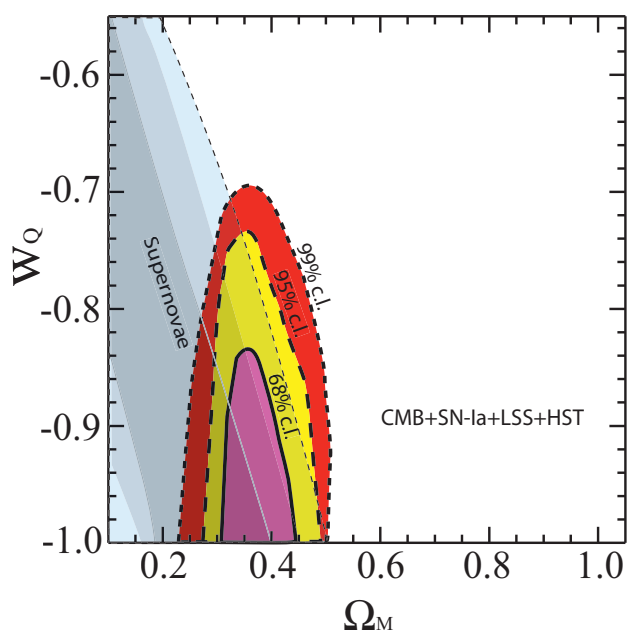

Figure 4. The likelihood contours in the $\left(\Omega_{M}, w_{Q}\right)$ plane, with the remaining parameters taking their best-fitting values for the joint $\mathrm{CMB}+\mathrm{SN}-\mathrm{Ia}+\mathrm{LSS}$ analysis described in the text. The contours correspond to $0.32,0.05$ and 0.01 of the peak value of the likelihood, which are the $68 \%, 95 \%$ and $99 \%$ confidence levels respectively.

We have provided new constraints on the dark energy by combining different cosmological data. We have examined BBN abundances in a cosmological scenario with a scaling field. We have quantitatively discussed how large values of the fractional density in the scaling field $\Omega_{Q}$ at $T \sim 1 \mathrm{MeV}$ can be in agreement with the observed values of ${ }^{4} \mathrm{He}$ and $D$, assuming standard Big Bang Nucleosynthesis. The $2 \sigma$ limit $\Omega_{Q}(1 \mathrm{MeV})<0.045$ severely constrains a wide class of quintessential scenarios, like those based on an exponential potential. For example, for the pure exponential potential the total energy today is restricted to $\Omega_{Q}=\frac{3}{4} \Omega_{Q}(1 \mathrm{MeV}) \leq 0.04$. This result put strong constraints on the presence of quintessence during recombination.

We have then provided constraints on the equation of state parameter $w_{Q}$. The new CMB results provided by Boomerang and DASI improve the constraints from previous and similar analysis (see e.g., [20], 228]), with $w_{Q}<-0.85$ at $68 \%$ c.l. $\left(w_{Q}<-0.76\right.$ at $95 \%$ c.l.). We have also demonstrated how the combination of $\mathrm{CMB}$ data with other datasets is crucial in order to break the $\Omega_{Q}-w_{Q}$ degeneracy. The constraints from each single datasets are, as expected, quite broad but compatible with each other, providing an important consistency test. When comparison is possible (i.e. restricting to similar priors and datasets), our analysis is compatible with other recent analysis on $w_{Q}([30])$. Our final result is perfectly in agreement with the $w_{Q}=-1$ cosmological constant case and gives no support to a quintessential field scenario with $w_{Q}>-1$. A frustrated network of domain walls or a purely exponential scaling field are excluded at high significance. In addition a number of quintessential models are highly disfavored, e.g. power law potentials with $p \geq 1$ and the oscillatory potential, to name a few.

It will be the duty of higher redshift datasets, for example from clustering observations [29] to point to a variation in $w$ that might place quintessence in a more favorable light.

The result obtained here, however, could be plagued by some of the theoretical assumptions we made. The CMB and LSS constraints can be weakened by the inclusion of additional relativistic degrees of freedom [33], by a background of gravity waves, of isocurvature perturbations or by adding features in the primordial perturbation spectra. These modifications are not expected in the most basic and simplified inflationary scenario but they are still compatible with the present data. The SN-Ia result has been obtained under the assumption of a constant-with-time $w_{Q}>-1$. Inclusion of the region with $w_{Q}<-1$ could affect our constraints (32]). In [24] we have shown that in general $w_{e f f}$ is a rather good approximation for dynamical quintessential models since the luminosity distance depends on $w_{Q}$ through a multiple integral that smears its redshift dependence, and our results are therefore valid for a wide class of quintessential models. This 'numbing' of sensitivity to $w_{Q}$, first noticed by [31], implies that maybe an effective equation of state is the most tangible parameter able to be extracted from supernovae. However with the promise of large data sets from Planck and SNAP satellites, opportunities may yet still be open to reconstruct a time varying equation of state [22].

Acknowledgements It is a pleasure to thank Ruth Durrer, Pedro Ferreira, Massimo Hansen and Matts Roos for comments and suggestions. RB and AM are supported by PPARC. SHH is supported by a Marie Curie Fellowship of the European Community under the contract HPMFCT-2000-00607. We acknowledge the use of CMBFAST [26].

\section{REFERENCES}

1. P.M. Garnavich et al, Ap.J. Letters 493, L5357 (1998); S. Perlmutter et al, Ap. J. 483, 565 (1997); S. Perlmutter et al (The Supernova Cosmology Project), Nature 39151 (1998); A.G. Riess et al, Ap. J. 116, 1009 (1998); B.P. Schmidt, Ap. J. 507, 46-63 (1998).

2. N. Bahcall, J. P. Ostriker, S. Perlmutter and 
P. J. Steinhardt, Science 284, 1481 (1999) astroph/9906463|; A. H. Jaffe et al,Phys. Rev. Lett. 86 (2001) astro-ph/0007333.

3. I. Zlatev, L. Wang, \& P. Steinhardt, Phys. Rev. Lett. 82 896-899 (1999).

4. L. Kawano, Fermilab-Pub-92/04-A (1992).

5. K.A. Olive and G. Steigman, Astrophys. J. Suppl. Ser. 97, 49 (1995).

6. Y.I. Izotov and T.X. Thuan, ApJ, 500188 (1998);

7. S. Burles and D. Tytler, ApJ, 499689 (1998).

8. P. G. Ferreira and M. Joyce, Phys.Rev.Lett. 79 (1997) 4740-4743 astro-ph/9707286.

9. S. Burles, K. M. Nollett, J. N. Truran and M. S. Turner, Phys. Rev. Lett. 82, 4176 (1999) astro-ph/9901157; S. Esposito, G. Mangano, A. Melchiorri, G. Miele and O. Pisanti, Phys. Rev. D 63 (2001) 043004 arXiv:astro$\mathrm{ph} / 0007419$;

10. S. H. Hansen, G. Mangano, A. Melchiorri, G. Miele and O. Pisanti, arXiv:astro$\mathrm{ph} / 0105385$.

11. P. Brax, J. Martin \& A. Riazuelo, Phys. Rev. D.,62 103505 (2000).

12. C Wetterich, Nucl. Phys B. 302668 (1988)

13. B. Ratra and J. Peebles, Phys. Rev D37 (1988) 321.

14. J. Frieman, C. Hill, A. Stebbins, I. Waga, Phys. Rev. Lett, 752077 (1995).

15. P. Ferreira and M. Joyce, Phys.Rev. D58 (1998) 023503.

16. C.B. Netterfield et al., astro-ph/0104460.

17. C. Pryke et al., astro-ph/0104489.

18. A. Lee et al., astro-ph/0104459.

19. Freedman W. L. et al, 2000, ApJ in press, preprint astro-ph/0012376.

20. S. Perlmutter, M.S. Turner, M. White, Phys.Rev.Lett. 83 670-673 (1999).

21. W. Hu, astro-ph/9801234.

22. J. Weller, A. Albrecht, Phys.Rev.Lett. 86 1939 (2001) astro-ph/0008314; D. Huterer and M. S. Turner, [astro-ph/0012510]; M. Tegmark, astro-ph/0101354.

23. R. Bean, S. H. Hansen and A. Melchiorri, Phys. Rev. D 64 (2001) 103508 arXiv:astro$\mathrm{ph} / 0104162$.

24. R. Bean and A. Melchiorri, arXiv:astroph/0110472, Phys. Rev. D in press.

25. G. Efstathiou \& J.R. Bond astro-ph/9807103;

A. Melchiorri \& L. M. Griffiths, New Astronomy Reviews, 45, 4-5, 2001, astro-ph/0011197.

26. M. Zaldarriaga \& U. Seljak, ApJ. 469437 (1996).

27. A. Melchiorri et al., Astrophys.J. 536 (2000) L63-L66, astro-ph/9911445.

28. J. R. Bond et al. [The MaxiBoom Collaboration], astro-ph/0011379.

29. M.O. Calvão, J.R.T De Mello Neto\& I. Waga [astro-ph/0 107029], J. Newman, C. Marinoni, A. Coil \& M. Davis astro-ph/0109131, T. Matsubara \& A. Szalay astro-ph/0105493].

30. P.S. Corasaniti \& E.J. Copeland, astroph/0107378; T. Saini, S. Raychaudhury, V. Sahni and A.A. Starobinsky, Phys. Rev. Lett. 85, 1162 - 1165 (2000); Y. Wang, G. Lovelace, astro-ph/0109233; D. Marfatia, V. Barger, astroph/0009256. C. Baccigalupi, A. Balbi, S. Matarrese, F. Perrotta, N. Vittorio, astro-ph/0109907.

31. I. Maor, R. Brustein, P.J. Steinhardt, Phys.Rev.Lett. 86 (2001) 6

32. I. Maor, R. Brustein, J. McMahon and P. J. Steinhardt, astro-ph/0112526

33. R. Bowen et al., arXiv:astro-ph/0110636. 